\title{
TAUBERIAN $L^{1}$-CONVERGENCE CLASSES OF FOURIER SERIES. I
}

BY

\author{
WILLIAM O. BRAY AND ČASLAV V. STANOJEVIC
}

\begin{abstract}
It is shown that the Stanojevic [2] necessary and sufficient conditions for $L^{1}$-convergence of Fourier series of $f \in L^{1}(T)$ can be reduced to the classical form. A number of corollaries of a recent Tauberian theorem are obtained for the subclasses of the class of Fourier coefficients satisfying $n^{\alpha}|\Delta \hat{f}(n)|=o(1)(n \rightarrow \infty)$ for some $0<\alpha \leqslant \frac{1}{2}$. For Fourier series with coefficients asymptotically even with respect to a sequence $\left\{l_{n}\right\}, l_{n}=o(n)(n \rightarrow \infty)$, and satisfying

$$
l_{n}^{-1 / q}\left(\sum_{k=n}^{n+\left[n / l_{n}\right]} k^{p-1}|\Delta \hat{f}(k)|^{p}\right)^{1 / p}=o(1) \quad(n \rightarrow \infty), \quad 1 / p+1 / q=1,
$$

necessary and sufficient conditions for $L^{1}$-convergence are obtained. In particular for $l_{n}=\left[\left\|\sigma_{n}(f)-f\right\|^{-1}\right]$, an important corollary is obtained which connects smoothness of $f$ with smoothness of $\{\hat{f}(n)\}$.
\end{abstract}

1. Comments on a recent Tauberian theorem. It is well known that the Banach space $L^{1}(T)$ of all complex valued Lebesgue integrable functions on $T=\mathbf{R} / 2 \pi \mathbf{Z}$ does not admit convergence in norm. That is to say there exists $f \in L^{1}(T)$ such that the partial sums of the Fourier series of $f$,

$$
S_{n}(f)=S_{n}(f, t)=\sum_{|k| \leqslant n} \hat{f}(k) e^{i k t}, \quad n=0,1,2, \ldots,
$$

do not converge in $L^{1}(T)$-norm (denoted $\|\cdot\|$ ) as $n \rightarrow \infty$. To study this situation many authors have defined, through the use of certain regularity and/or speed conditions on the sequence $\{\hat{f}(n)\}$, subclasses (called $L^{1}$-convergence classes) of Fourier coefficients for which

$$
\begin{aligned}
\left\|S_{n}(f)-f\right\| & =o(1) \quad(n \rightarrow \infty) \\
& \text { if and only if } \hat{f}(n) \lg |n|=o(1) \quad(|n| \rightarrow \infty),
\end{aligned}
$$

(see [1] for a survey). All classical results in this vein were formulated separately for cosine series, $a_{0} / 2+\sum_{n=1}^{\infty} a_{n} \cos n x$, and sine series, $\sum_{n=1}^{\infty} b_{n} \sin n x$. These correspond formally in the complex form to even coefficients $(\hat{f}(n)=\hat{f}(-n))$ and odd coefficients $(\hat{f}(n)=-\hat{f}(-n))$, respectively.

Received by the editors August 10, 1981.

1980 Mathematics Subject Classification. Primary 42A20, 42A32.

Key words and phrases. $L^{\prime}$-convergence of Fourier series. 
The approach to the problem here leads to the introduction of asymptotically even coefficients, i.e., a function $f \in L^{1}(T)$ is said to belong to the class $Q \mathcal{E}$ if the Fourier coefficients of $f$ satisfy

$$
\begin{gathered}
\frac{1}{n} \sum_{k=1}^{n}|\hat{f}(k)-\hat{f}(-k)| \lg \dot{k}=o(1) \quad(n \rightarrow \infty), \\
\lim _{\lambda \searrow 1} \varlimsup_{n} \sum_{k=n}^{[\lambda n]}|\Delta(\hat{f}(k)-\hat{f}(-k))| \lg k=0 .
\end{gathered}
$$

Inasmuch as the difference $\hat{f}(n)-\hat{f}(-n)$ is a constant multiple of the $n$th sine Fourier coefficients of $f$, our approach can be seen to mimic the difference between analytical methods applied to cosine and sine series classically. See $\$ 3$ for further comments regarding this.

A reformulation of a recent Tauberian theorem of Stanojević [2] generalizing many classical results is the following

THEOREM 1.1. Let

$$
S(f) \sim \sum_{|n|<\infty} \hat{f}(n) e^{i n t}
$$

be the Fourier series of $f \in \mathbb{Q E}$. If for some $1<p \leqslant 2$

$$
\lim _{\lambda \searrow 1} \varlimsup_{n \rightarrow \infty} \sum_{k=n}^{[\lambda n]} k^{p-1}|\Delta \hat{f}(k)|^{p}=0,
$$

then $(\mathrm{Y})$ holds.

The original version of this result gave the necessary and sufficient condition in the form

$$
\left\|\hat{f}(n) E_{n}+\hat{f}(-n) E_{-n}\right\|=o(1) \quad(n \rightarrow \infty),
$$

where $E_{n}=E_{n}(t)=\sum_{k=0}^{n} e^{i k t}$. That (ST) is equivalent with $\hat{f}(n) \lg |n|=o(1)(|n| \rightarrow$ $\infty)$ follows from the general lemma below needed in $\$ 2$.

LEMMA 1.1. Let $\left\{C_{n}\right\}_{|n| \rightarrow \infty} \subset \mathbf{C}$ and let $\left\{l_{n}\right\}_{1}^{\infty} \subset \mathbf{R}^{+}$such that $l_{n}=o(n)(n \rightarrow \infty)$. Let $T_{n}=\left(l_{n} \pi / n, 2 \pi-l_{n} \pi / n\right)$. Then

$$
J_{n}=\int_{T_{n}}\left|C_{n} E_{n}(t)+C_{-n} E_{-n}(t)\right| d t=o(1) \quad(n \rightarrow \infty)
$$

if and only if $C_{n} \lg \left(|n| / l_{|n|}\right)=o(1)(|n| \rightarrow \infty)$.

Proof. Note that

$$
\begin{aligned}
J_{n} & =\int_{l_{n} \pi / n}^{\pi}\left|C_{n} E_{n}(t)+C_{-n} E_{-n}(t)\right| d t+\int_{-\pi}^{-l_{n} \pi / n}\left|C_{n} E_{n}(t)+C_{-n} E_{-n}(t)\right| d t \\
& =\int_{l_{n} \pi / n}^{\pi}\left\{\left|C_{n} E_{n}(t)+C_{-n} E_{-n}(t)\right|+\left|C_{n} E_{-n}(t)+C_{-n} E_{n}(t)\right|\right\} d t \\
& \geqslant\left|C_{n}+C_{-n}\right| \int_{l_{n} \pi / n}^{\pi}\left|E_{n}(t)+E_{-n}(t)\right| d t \\
& =\left|C_{n}+C_{-n}\right| \int_{l_{n} \pi / n}^{\pi}\left|D_{n}(t)\right| d t+o(1) \quad(n \rightarrow \infty),
\end{aligned}
$$


where $D_{n}(t)=\sin \left(n+\frac{1}{2}\right) t / \sin \frac{t}{2}$ is the Dirichlet kernel. Elementary calculation will show that

$$
\int_{l_{n} \pi / n}^{\pi}\left|D_{n}(t)\right| d t \geqslant M \lg \frac{n}{l_{n}}+O(1) \quad(n \rightarrow \infty)
$$

where $M$ is an absolute constant. Hence, $J_{n} \geqslant M\left|C_{n}+C_{-n}\right| \lg \left(n / l_{n}\right)+o(1)(n \rightarrow$ $\infty)$, from which it follows that $\left|C_{n}+C_{-n}\right| \lg \left(n / l_{|n|}\right)=o(1)$. Now,

$$
\begin{aligned}
J_{n} & =\int_{T_{n}}\left|\left(C_{n}+C_{-n}\right) E_{n}(t)+C_{-n}\left(E_{-n}(t)-E_{n}(t)\right)\right| d t \\
& \geqslant|| C_{n}+C_{-n}\left|\int_{T_{n}}\right| E_{n}(t)|d t-| C_{-n}\left|\int_{T_{n}}\right| E_{n}(t)-E_{-n}(t)|d t| .
\end{aligned}
$$

Calculations similar to these above will show that both the integrals on the right-hand side of the above inequality are on the order of a constant times $\lg \left(n / l_{|n|}\right)$ from which the result follows.

Observing that (ST) is equivalent with

$$
\int_{T_{n}^{\prime}}\left|\hat{f}(n) E_{n}(t)+\hat{f}(-n) E_{-n}(t)\right| d t=o(1) \quad(n \rightarrow \infty),
$$

where $T_{n}^{\prime}=\left(\frac{\pi}{n}, 2 \pi-\frac{\pi}{n}\right)$, choosing $l_{n}=1$ for all $n$ in the above lemma gives that (ST) is equivalent with $\hat{f}(n) \lg |n|=o(1)(|n| \rightarrow \infty)$.

Theorem 1.1 has the following corollary which indicates that (HK) is a way of controlling subsequent behavior of the sequence $\{\hat{f}(n)\}$.

CorollaRY 1.1. Let $S(f)$ be as in Theorem 1.1. If $n \Delta \hat{f}(n)=O(1)(n \rightarrow \infty)$ then (Y) holds.

In lieu of the fact that almost all known $L^{1}$-convergence classes are proper subclasses of all Fourier coefficients satisfying

(BOX)

$$
n^{\alpha}|\Delta \hat{f}(n)|=o(1) \quad(n \rightarrow \infty) \quad \text { for some } 0<\alpha \leqslant \frac{1}{2},
$$

the purpose of this paper is to provide certain classes of Fourier coefficients for which (BOX) is an $L^{1}$-convergence class. The question of whether (BOX) in general is an $L^{1}$-convergence class remains open. The following examples obtained by decomposing (HK) give special cases in which (BOX) is an $L^{1}$-convergence class.

COROllary 1.2. Let

$$
S(f) \sim \sum_{|n|<\infty} \hat{f}(n) e^{i n t}
$$

be the Fourier series of $f \in Q \mathcal{E}$ and let for some $1<p \leqslant 2$ and some $\lambda>1\left(\frac{1}{p}+\frac{1}{q}=1\right)$,

$$
\left(\sum_{k=n}^{[\lambda n]} k^{p-1}|\Delta \hat{f}(k)|^{p}\right)^{1 / p}=O\left(n^{1 / q}|\Delta \hat{f}(n)|\right) \quad(n \rightarrow \infty) .
$$

If $\{\hat{f}(n)\}$ satisfies (BOX) with $\alpha=\frac{1}{q}$, then $(\mathrm{Y})$ holds.

From (1.1) and (BOX) with $\alpha=\frac{1}{q}$ it follows that $\{\hat{f}(n)\}$ satisfies (HK). 
Clearly Corollary 1.2 is rather naive; however in the light of forthcoming examples it and the following more sophisticated results are significant. For $\lambda>1$ and $f \in L^{1}(T)$, define $\mu_{n}(f, \lambda)$ to be the number of nonzero $\Delta \hat{f}(k)$ for $n \leqslant k \leqslant[\lambda n]$.

Corollary 1.3. Let $S(f)$ be as in Corollary 1.2. If for some $1<p \leqslant 2$,

$$
\lim _{\lambda \supset 1} \varlimsup_{n \rightarrow \infty} \mu_{n}^{1 / p}(f, \lambda) \max _{n \leqslant k \leqslant[\lambda n]} k^{1 / q}|\Delta \hat{f}(k)|=0,
$$

then (Y) holds.

The proof is immediate from the inequality:

$$
\left(\sum_{k=n}^{[\lambda n]} k^{p-1}|\Delta \hat{f}(k)|^{p}\right)^{1 / p} \leqslant \mu_{n}^{1 / p}(f, \lambda) \max _{n \leqslant k \leqslant[\lambda n]} k^{1 / q}|\Delta \hat{f}(k)| .
$$

Observe that Corollary 1.1 is also a corollary to this result. This follows from the inequalities

$$
\begin{aligned}
\mu_{n}^{1 / p}(f, \lambda) \max _{n \leqslant k \leqslant[\lambda n]} k^{1 / q}|\Delta \hat{f}(k)| & \leqslant\left(\frac{\mu_{n}(f, \lambda)}{n}\right)^{1 / p} \lambda^{1 / p} \max _{n \leqslant k \leqslant[\lambda n]} k|\Delta \hat{f}(k)| \\
& \leqslant\left((\lambda-1)+\frac{1}{n}\right)^{1 / p} \lambda^{1 / p} \max _{n \leqslant k \leqslant[\lambda n]} k|\Delta \hat{f}(k)| .
\end{aligned}
$$

An interesting example [3] is found by considering the cosine series $a_{0} / 2+$ $\sum_{n=1}^{\infty} a_{n} \cos n x$ where the sequence $\left\{a_{n}\right\}$ is defined as follows. For $1<p \leqslant 2$ and $\frac{1}{q}<s<1$, let $\Delta a_{l}=1 / 2^{s n}$ if $l=2^{n}, n=0,1,2, \ldots$, and let $\Delta a_{l}=0$ otherwise. Then Fomin has shown that the above series is a Fourier series. More generally one can consider Fourier series whose coefficients satisfy $\Delta a_{l}=0$ unless $l=n_{m}, m=$ $0,1,2, \ldots$, where $\left\{n_{m}\right\}$ is lacunary or satisfies the condition $\sum_{m=k}^{\infty} n_{m}^{-1}=O\left(n_{k}^{-1}\right)$ $(k \rightarrow \infty)$ (see [4] for properties of sequences satisfying this latter condition).

As a third approach to (BOX) consider the following.

Corollary 1.4. Let $S(f)$ be as in Corollary 1.2 and let for some $1<p \leqslant 2$,

$$
\varlimsup_{n \rightarrow \infty}\left(\sum_{k=n}^{[\lambda n]}\left(k^{p-1}|\Delta \hat{f}(k)|^{p}\right)^{1 / 2}\right)^{1 / p}=O(1) \quad(\lambda \searrow 1) .
$$

If $\{\hat{f}(n)\}$ satisfies $(\mathrm{BOX})$ with $\alpha=\frac{1}{q}$, then $(\mathrm{Y})$ holds.

The proof of this is done through inequalities:

$$
\begin{aligned}
& \left(\sum_{k=n}^{[\lambda n]} k^{p-1}|\Delta \hat{f}(k)|^{p}\right)^{1 / p} \\
& \quad \leqslant\left(\max _{n \leqslant k<[\lambda n]} k^{1 / q}|\Delta \hat{f}(k)|\right)^{1 / 2}\left(\sum_{k=n}^{[\lambda n]} k^{(p-1) / 2}|\Delta \hat{f}(k)|^{p / 2}\right)^{1 / p} \\
& \leqslant M\left(\max _{n \leqslant k \leqslant[\lambda n]} k^{1 / q}|\Delta f(k)|\right)^{1 / 2},
\end{aligned}
$$

where $M$ is the constant stemming from (1.3). 
Corollary 1.5. Let $S(f)$ be as in Corollary 1.2 and let for some $1<p \leqslant 2$

$$
\lim _{n \rightarrow \infty}\left(\sum_{k=n}^{[\lambda n]} k^{(p-1) / \lambda}|\Delta f(k)|^{p / \lambda}\right)^{1 / p}=O(1) \quad(\lambda \searrow 1) .
$$

If $\{\hat{f}(n)\}$ satisfies (BOX) with $\alpha=\frac{1}{q}$, then $(\mathrm{Y})$ holds.

This follows from an inequality very much like (1.4). As a final decomposition, let $\lambda=q-1$ in Corollary 1.5 , i.e.

Corollary 1.6. Let $S(F)$ be as in Corollary 1.2 and let

$$
\varlimsup_{n \rightarrow \infty}\left(\sum_{k=n}^{[(q-1) n]} k^{(p-1)^{2}} \mid \Delta f(k) p^{p(p-1)}\right)^{1 / p}=O(1) \quad(q \downarrow 2) .
$$

If $n^{1 / 2} \Delta \hat{f}(n)=o(1)(n \rightarrow \infty)$ then $(\mathrm{Y})$ holds.

The main result of $\$ 2$ (Theorem 2.1) and especially one corollary of it will provide deeper insight into the following result [2].

THEOREM 1.2. Let

$$
S(f) \sim \sum_{|n|<\infty} \hat{f}(n) e^{i n t}
$$

be the Fourier series of $f \in L^{1}(T)$ with even coefficients such that $\left\|\sigma_{n}(f)-f\right\| \lg n=$ $o(1)(n \rightarrow \infty)$. If for some $1<p \leqslant 2$

$$
\left(\frac{1}{n} \sum_{k=n}^{n+[n / \lg n]}|\hat{f}(k)|^{p}\right)^{1 / p} \lg n=o(1) \quad(n \rightarrow \infty)
$$

and

$$
n[\lg n]^{-1 / p} \max _{n \leqslant k \leqslant n+[n / \lg n]}|\Delta \hat{f}(k)|=o(1)(n \rightarrow \infty),
$$

then $\left\|S_{n}(f)-f\right\|=o(1)(n \rightarrow \infty)$ if and only if $\hat{f}(n) \lg n=o(1)(n \rightarrow \infty)$.

Our results of $\$ 2$ not only generalize Theorem 1.2 but in the light of Corollaries 1.2-1.6 given an intrinsic scope into the nature of $L^{1}$-convergence classes and their relation to subclasses of $L^{1}(T)$ defined through conditions on the integral modulus of continuity.

2. An alternative Tauberian theorem. Let $f \in L^{1}(T)$ and let $\left\{\phi_{n} \mathrm{j}\right\}$ be a sequence of natural numbers tending to infinity with $n$; then the de la Valle Poussin sums of the Fourier series of $f$ are given by:

$$
V_{n+\phi_{n}}(f)=V_{n+\phi_{n}}(f, t)=\frac{1}{\phi_{n}+1} \sum_{k=n}^{n+\phi_{n}} S_{k}(f, t), \quad n=0,1,2, \ldots
$$

To these correspond a natural estimate.

LEMMA 2.1. $\left\|V_{n+\phi_{n}}(f)-f\right\| \leqslant M\left\|\sigma_{n}(f)-f\right\|\left(n / \phi_{n}\right)^{1 / 2}+o(1)(n \rightarrow \infty)$, where $M$ is an absolute constant. 
Proof. From the identity,

$$
V_{n+\phi_{n}}(f, t)-f(t)=\frac{1}{\phi_{n}+1} \sum_{k=n}^{n+\phi_{n}}\left(D_{k} *\left(f-\sigma_{n}(f)\right)(t)+\left(\sigma_{n}(f, t)-f(t)\right)\right),
$$

where "*" denotes convolution and $D_{n}(t)=\sin \left(n+\frac{1}{2}\right) t / \sin \frac{t}{2}$ is the Dirichlet kernel, Young's inequality yields

$$
\left\|V_{n+\phi_{n}}(f)-f\right\| \leqslant\left\|\sigma_{n}(f)-f\right\|\left\|\frac{1}{\phi_{n}+1} \sum_{k=n}^{n+\phi_{n}} D_{k}\right\|+o(1) \quad(n \rightarrow \infty) .
$$

The proof will be completed by showing

$$
\tau_{n}=\left\|\frac{1}{\phi_{n}+1} \sum_{k=n}^{n+\phi_{n}} D_{k}\right\| \leqslant M\left(\frac{n}{\phi_{n}}\right)^{1 / 2}+O(1) \quad(n \rightarrow \infty) .
$$

For this, consider,

$$
\begin{aligned}
\tau_{n} & =\frac{1}{\pi\left(\phi_{n+1}\right)} \int_{0}^{\pi}\left|\sum_{k=n}^{n+\phi_{n}} D_{k}(t)\right| d t \\
& =\frac{1}{\pi\left(\phi_{n}+1\right)}\left\{\int_{0}^{\pi / n}+\int_{\pi / n}^{\pi}\right\}\left|\sum_{k=n}^{n+\phi_{n}} D_{k}(t)\right| d t=I_{1}+I_{2} .
\end{aligned}
$$

A uniform estimate on the Dirichlet kernel is applied to the first integral, i.e.,

$$
I_{1} \leqslant \frac{1}{\pi\left(\phi_{n}+1\right)} \cdot \frac{\pi}{n} \cdot\left(n+\phi_{n}+1\right)\left(\phi_{n}+1\right)=O(1) \quad(n \rightarrow \infty)
$$

The second integral is handled as follows:

$$
I_{2} \leqslant \frac{1}{\pi\left(\phi_{n}+1\right)}\left\{\int_{\pi / n}^{\pi} \frac{d t}{(\sin (t / 2))^{2}}\right\}^{1 / 2}\left\{\int_{\pi / n}^{\pi}\left|\sum_{k=n}^{n+\phi_{n}} \sin \left(k+\frac{1}{2}\right) t\right|^{2}\right\}^{1 / 2} \leqslant M\left(\frac{n}{\phi_{n}}\right)^{1 / 2},
$$

for an appropriate constant $M$. Combining the estimates gives the lemma.

The above proof is like that given in Fomin [5] who obtained the estimate (to which ours is a corollary),

$$
\left\|V_{n+\phi_{n}}(f)-f\right\| \leqslant M E_{n}(f)\left(n / \phi_{n}\right)^{1 / 2}+o(1) \quad(n \rightarrow \infty),
$$

where $E_{n}(f)=\inf \left\|T_{n}-f\right\|$, the inf taken over the class of all trigonometric polynomials $T_{n}=T_{n}(t)$ of order $n$. However the estimate involving the Fejér sums is more useful for later results and the proof is a special case of the Hölder-HausdorffYoung technique used in proving Theorem 1.1 and the results of this section.

For what follows, $\mathcal{L}$ denotes the class of all sequences $\left\{l_{n}\right\}$ of real numbers tending to infinity with $n$ and $l_{n}=o(n)(n \rightarrow \infty)$. Also, for $f \in L^{1}(T)$, define,

$$
\mathcal{L}(f)=\left\{\left\{l_{n}\right\} \in \mathcal{L} \mid\left\|\sigma_{n}(f)-f\right\| l_{n}^{1 / 2}=o(1)(n \rightarrow \infty)\right\}
$$

and

$$
\mathcal{G}(f)=\left\{\left\{l_{n}\right\} \in \mathcal{L} \mid\left\|\sigma_{n+\left[n / l_{n}\right]}(f)-\sigma_{n}(f)\right\| l_{n}=o(1)(n \rightarrow \infty)\right\} .
$$

Observe that $\mathcal{L}(f) \neq \varnothing$ for all $f \in L^{1}(T)$ and consequently we have two propositions. 
Proposition 2.1. If $\left\{l_{n}\right\} \in \mathcal{L}(f)$, then $\left\|V_{n+\left[n / l_{n}\right]}(f)-f\right\|=o(1)(n \rightarrow \infty)$.

Proposition 2.2. $\mathcal{L}(f) \subset \mathcal{G}(f)$.

Proof. This inclusion follows from the identity,

$$
\begin{aligned}
V_{n+\left[n / l_{n}\right]}(f, t) & -\sigma_{n}(f, t) \\
= & \frac{n+\left[n / l_{n}\right]}{\left[n / l_{n}\right]+1}\left(\sigma_{n+\left[n / l_{n}\right]}(f, t)-\sigma_{n}(f, t)\right)-\frac{\sigma_{n}(f, t)}{\left[n / l_{n}\right]+1} .
\end{aligned}
$$

Before presenting the main results of this section we need to define another class of asymptotically even coefficients. A function $f \in L^{1}(T)$ is said to belong to the class $\mathscr{Q E} \mathcal{G}$ if for some $\left\{l_{n}\right\} \in \mathcal{G}(f)$,

$$
\begin{gathered}
{\left[\frac{n}{l_{n}}\right]^{-1} \sum_{k=n}^{n+\left[n / l_{n}\right]}|\hat{f}(k)-\hat{f}(-k)| \lg k=o(1) \quad(n \rightarrow \infty),} \\
\sum_{k=n}^{n+\left[n / l_{n}\right]}|\Delta(\hat{f}(k)-\hat{f}(-k))| \lg k=o(1) \quad(n \rightarrow \infty) .
\end{gathered}
$$

LemMa 2.2. Let $\left\{c_{n}\right\} \subset \mathrm{C}$, let $n>n$, and let $1<p \leqslant 2$. Then

$$
I_{n m}=\int_{\pi / m-n}^{\pi}\left|\sum_{k=n}^{m} c_{k} D_{k}(t)\right| d t \leqslant A_{p}(m-n)^{1 / q}\left(\sum_{k=n}^{m}\left|c_{k}\right|^{p}\right)^{1 / p},
$$

where $A_{p}$ is a constant dependent on $p$.

Proof. Applying successively the inequalities of Hölder and Hausdorff-Young one obtains,

$$
\begin{aligned}
I_{n m} & \leqslant\left\{\int_{\pi / m-n}^{\pi} \frac{d t}{(\sin (t / 2))^{p}}\right\}^{1 / p}\left\{\int_{\pi / m-n}^{\pi}\left|\sum_{k=n}^{m} c_{k} \sin \left(k+\frac{1}{2}\right) t\right|^{q} d t\right\}^{1 / q} \\
& \leqslant A_{p}(m-n)^{1 / q}\left\|\sum_{k=n}^{m} c_{k} \sin \left(k+\frac{1}{2}\right) t\right\|_{q} \leqslant A_{p}(m-n)^{1 / q}\left(\sum_{k=n}^{m}\left|c_{k}\right|^{p}\right)^{1 / p}
\end{aligned}
$$

where $\|\cdot\|_{q}$ denotes $L^{q}(T)$ norm.

THEOREM 2.1. Let

$$
S(f) \sim \sum_{|n|<\infty} \hat{f}(n) e^{i n t}
$$

be the Fourier series of $f \in \mathbb{Q E G}$. If for some $1<p \leqslant 2$,

$$
l_{n}^{-1 / q}\left(\sum_{k=n}^{n+\left[n / l_{n}\right]} k^{p-1}|\Delta \hat{f}(k)|^{p}\right)^{1 / p}=o(1) \quad(n \rightarrow \infty),
$$

then $\left\|S_{n}(f)-f\right\|=o(1)(n \rightarrow \infty)$ if and only if $\hat{f}(n) \lg \left(|n| / l_{|n|}\right)=o(1)(|n| \rightarrow \infty)$.

Proof. A well-known identity for $m>n$ is

$$
S_{n}(f, t)-\sigma_{n}(f, t)=\frac{m+1}{m-n}\left(\sigma_{m}(f, t)-\sigma_{n}(f, t)\right)-Q_{n}^{m}(f, t),
$$


where

$$
Q_{n}^{m}(f, t)=\sum_{|k|=n+1}^{m} \frac{m-|k|+1}{m-n} \hat{f}(k) e^{i k t}
$$

Since,

$$
I_{m n}(f)=\int_{-\pi / m-n}^{\pi / m-n}\left|Q_{n}^{m}(f, t)\right| d t \leqslant \frac{2 \pi}{m-n} \sum_{|k|=n+1}^{m}|\hat{f}(k)|,
$$

it follows that $I_{n m}(f)=o(1)$ when $n, m, m-n \rightarrow \infty$. Consequently, letting $m=n$ $+\left[n / l_{n}\right],\left\{l_{n}\right\}$ given by the hypothesis $f \in \mathcal{Q} \mathcal{E} \mathcal{G}$, one sees that $\left\|S_{n}(f)-\sigma_{n}(f)\right\|=$ $o(1)(n \rightarrow \infty)$ if and only if

$$
\int_{T_{n}}\left|Q_{n}(f, t)\right| d t=o(1) \quad(n \rightarrow \infty),
$$

where $Q_{n}(f, t)$ is $Q_{n}^{m}(f, t)$ after the above substitution, and

$$
T_{n}=\left(\frac{\pi}{\left[n / l_{n}\right]}, 2 \pi-\frac{\pi}{\left[n / l_{n}\right]}\right)
$$

Now, applying summation by parts to $Q_{n}^{m}(f, t)$ using the identity $D_{n}(t)=E_{n}(t)+$ $E_{n}(t)-1$, and rearranging yields the identity

$$
\begin{aligned}
Q_{n}^{m}(f, t)= & \sum_{k=n}^{m-1} \frac{m-k}{m-n} \Delta \hat{f}(k) D_{k}(t)-\sum_{k=n}^{m-1} \frac{m-k}{m-n} \Delta(\hat{f}(k)-\hat{f}(-k)) E_{-k}(t) \\
& +\sum_{k=n+1}^{m} \frac{\hat{f}(k)}{m-n} D_{k}(t)-\sum_{k=n+1}^{m} \frac{\hat{f}(k)-\hat{f}(-k)}{m-n} E_{-k}(t) \\
& -\left[\hat{f}(n) E_{n}(t)+\hat{f}(-n) E_{-n}(t)\right] .
\end{aligned}
$$

Integrating over $T_{n}$, using the estimate $\left\|E_{-k}\right\|=O(\lg k)(k \rightarrow \infty)$, and Lemma 2.2 gives a fundamental inequality:

$$
\begin{aligned}
& \left|\int_{T_{n}}\right| Q_{n}(f, t)\left|d t-I_{n}\left(f, l_{n}\right)\right| \\
& \leqslant A_{p}\left[\frac{n}{l_{n}}\right]^{-1 / q}\left(\sum_{k=n}^{n+\left[n / l_{n}\right]}|\Delta \hat{f}(k)|^{p}\right)^{1 / p}+B_{p}\left(\left[\frac{n}{l_{n}}\right]^{-1} \sum_{k=n}^{n+\left[n / l_{n}\right]}|\hat{f}(k)|^{p}\right)^{1 / p} \\
& \quad+A\left\{\sum_{k=n}^{n+\left[n / l_{n}\right]}|\Delta(\hat{f}(k)-\hat{f}(-k))| \lg k+\left[\frac{n}{l_{n}}\right]^{-1} \sum_{k=n}^{n+\left[n / l_{n}\right]}|\hat{f}(k)-\hat{f}(-k)| \lg k\right\},
\end{aligned}
$$

where $A_{p}, B_{p}$ and $A$ are constants and,

$$
I_{n}\left(f, l_{n}\right)=\int_{T_{n}}\left|\hat{f}(n) E_{n}(t)+\hat{f}(-n) E_{-n}(t)\right| d t
$$


Letting $n \rightarrow \infty$, the third and fourth terms on the right-hand side are $o(1)$ since $f \in \mathbb{Q} \mathcal{E} \mathcal{G}$, and the second from the fact that $\hat{f}(n)=o(1)(n \rightarrow \infty)$. Hence

$$
\begin{aligned}
& \left|\int_{T_{n}}\right| Q_{n}(f, t)\left|d t-I_{n}\left(f, l_{n}\right)\right| \\
& \quad \leqslant A_{p} l_{n}^{-1 / q}\left(\sum_{k=n}^{n+\left[n / l_{n}\right]} k^{p-1}|\Delta \hat{f}(k)|^{p}\right)^{1 / p}+o(1) \quad(n \rightarrow \infty) .
\end{aligned}
$$

From (KL), it follows that (2.2) holds if and only if $I_{n}\left(f, l_{n}\right)=o(1)(n \rightarrow \infty)$ which by Lemma 1.1 is equivalent with $\hat{f}(n) \lg \left(|n| / l_{|n|}\right)=o(1)(|n| \rightarrow \infty)$. This concludes the proof.

COROllary 2.1.1. Let

$$
S(f) \sim \sum_{|n|<\infty} \hat{f}(n) e^{i n t}
$$

be the Fourier series of $f \in L^{1}(T)$ such that (AE1) holds for $l_{n}=\left\|\sigma_{n}(f)-f\right\|^{-1}$, and let

$$
(n \lg n)\left\|\sigma_{n}(f)-f\right\| \Delta(\hat{f}(n)-\hat{f}(-n))=O(1) \quad(n \rightarrow \infty)
$$

If

$$
n \Delta \hat{f}(n)\left\|\sigma_{n}(f)-f\right\|=O(1) \quad(n \rightarrow \infty),
$$

then $\left\|S_{n}(f)-f\right\|=o(1)$ if and only if $\hat{f}(n) \lg \left(|n|\left\|\sigma_{n}(f)-f\right\|\right)=o(1), n \rightarrow \infty$.

Proof. Let $l_{n}=\left[\left\|\sigma_{n}(f)-f\right\|^{-1}\right]$; then $\left\{l_{n}\right\} \in \mathcal{L}(f)$. From the inequalities

$$
\sum_{k=n}^{n+\left[n / l_{n}\right]}|\Delta(\hat{f}(k)-\hat{f}(-k))| \lg k \leqslant\left[\frac{n}{l_{n}}\right] \max _{n \leqslant k \leqslant n+\left[n / l_{n}\right]}|\Delta(\hat{f}(k)-\hat{f}(-k))| \lg k
$$

and

$$
l_{n}^{-1 / q}\left(\sum_{k=n}^{n+\left[n / l_{n}\right]} k^{p-1}|\Delta \hat{f}(k)|\right)^{1 / p} \leqslant M\left(\frac{n}{l_{n}}\right)_{n \leqslant k \leqslant n+\left[n / l_{n}\right]}|\Delta \hat{f}(k)|,
$$

where $M$ is a constant, the result follows.

Let $f \in L^{1}(T)$ and let $\omega^{*}(f, \delta), \delta>0$, be the integral modulus of continuity of $f$, i.e.,

$$
\omega^{*}(f ; \delta)=\sup _{|n| \leqslant \delta}\|f(\cdot+h)+f(\cdot-h)-2 f(\cdot)\| .
$$

Then a well-known inequality from approximation theory is (see [6, p. 77]),

$$
\left\|\sigma_{n}(f)-f\right\|=O\left(\omega^{*}\left(f ; n^{-1 / 2}\right)\right) .
$$

Thus, (2.3) and (2.4) are implied by

$$
(n \lg n) \omega^{*}\left(f ; n^{-1 / 2}\right)|\Delta(\hat{f}(n)-\hat{f}(-n))|=o(1) \quad(n \rightarrow \infty)
$$

and

$$
n \Delta \hat{f}(n) \omega^{*}\left(f ; n^{-1 / 2}\right)=o(1) \quad(n \rightarrow \infty),
$$


respectively. From this, conditions (2.3) and (2.4) represent a blending of smoothness conditions on the function $f$ and its Fourier transform $\{\hat{f}(n)\}$. A situation to exemplify this would be to let $f$ satisfy a Lipschitz condition $\left(\omega^{*}(f ; \delta)=O\left(\delta^{\alpha}\right)\right.$, $0<\alpha \leqslant 1)$.

A way of judging the strength of the corollaries of Theorems 1.1 and 2.1 is to check whether they imply Corollary 1.1 which in a Tauberian sense is best possible.

COROllary 2.1.2. Let

$$
S(f) \sim \sum_{|n|<\infty} \hat{f}(n) e^{i n t}
$$

be the Fourier series of $f \in \mathbb{Q G}$. If $n \Delta \hat{f}(n)=O(1)(n \rightarrow \infty)$ then $(\mathrm{Y})$ holds.

One further corollary to Theorem 2.1 is the following result which is an analogue of a result of Fomin [5] for real valued functions.

Corollary 2.1.3. Let $S(f) \sim \Sigma_{|n|<\infty} \hat{f}(n) e^{i n t}$ be the Fourier series of $f \in L^{1}(T)$ such that for all $\left\{l_{n}\right\} \in \mathcal{L},(\mathrm{AE} 1)$ and (AE2) hold. If for every $\left\{l_{n}\right\} \in \mathcal{L}$ condition (KL) holds, then (Y) holds.

As a final result of this section, the following theorem gives an indication of the generality of Theorem 2.1 .

THEOREM 2.2. Let

$$
S(f) \sim \sum_{|n|<\infty} \hat{f}(n) e^{i n t}
$$

be the Fourier series of $f \in L^{1}(T)$ such that, for some $\left\{l_{n}\right\} \in \mathcal{L}$, conditions (AE1) and (AE2) hold. If, for some $1<p \leqslant 2$, condition (KL) holds and $\hat{f}(n) \lg \left(|n| / l_{|n|}\right)=o(1)$ $(|n| \rightarrow \infty)$, then $\left\|S_{n}(f)-f\right\|=o(1)(n \rightarrow \infty)$ if and only if $\left\{l_{n}\right\} \in \mathcal{G}(f)$.

Proof. Identity (2.1) can be rewritten as

$$
\left(S_{n}(f, t)-\sigma_{n}(f, t)\right)-\frac{m+1}{m-n}\left(\sigma_{m}(f, t)-\sigma_{n}(f, t)\right)=-Q_{n}^{m}(f, t) .
$$

Making the substitution $m=n+\left[n / l_{n}\right]$, the techniques of Theorem 2.1 give the inequality:

$$
\begin{aligned}
& \left|\left\|S_{n}(f)-\sigma_{n}(f)\right\|-\frac{n+\left[n / l_{n}\right]+1}{\left[n / l_{n}\right]+1}\left\|\sigma_{n+\left[n / l_{n}\right]}(f)-\sigma_{n}(f)\right\|\right| \\
& \leqslant A_{p} l_{n}^{-1 / q}\left(\sum_{k=n}^{n+\left[n / l_{n}\right]} k^{p-1}|\Delta \hat{f}(k)|^{p}\right)^{1 / p}+B_{p}\left[\frac{n}{l_{n}}\right]^{-1}\left(\sum_{k=n}^{n+\left[n / l_{n}\right]}|\hat{f}(k)|^{p}\right)^{1 / p} \\
& \quad+A\left[\sum_{k=n}^{n+\left[n / l_{n}\right]}|\Delta(\hat{f}(k)-\hat{f}(-k))| \lg k+\left[\frac{n}{l_{n}}\right]^{-1} \sum_{k=n}^{n+\left[n / l_{n}\right]}|\hat{f}(k)-\hat{f}(-k)| \lg k\right] \\
& \quad+I_{n}\left(f, l_{n}\right),
\end{aligned}
$$

from which the result follows. 
3. Further comments. The results of $\S 3$ clearly indicate that in order to define classes of Fourier coefficients such that $(\mathrm{Y})$ holds, both conditions of smoothness on the function $f$ and its Fourier transform $\{\hat{f}(n)\}$ are required. An interesting direction for further work would be to consider the classical results on the $L^{1}$-convergence problem in the vein of conditions of smoothness on $f$.

Another interesting problem would be to prove Corollary 1.1 or even Corollary 2.1.1 without resorting to the Hölder-Hausdorff-Young technique used above. This would shed greater insight into the $L^{1}$-convergence problem and also into the aforementioned blending of smoothness conditions.

As mentioned in $\$ 1$, conditions of asymptotically even type are sort of mimicking the different analytical techniques used classically to attack the $L^{1}$-convergence problem separately for cosine and sine series. Since the time of their introduction, it has occurred to the authors that the above theory can be developed without the direct notion of asymptotically even as above. This is one purpose of the forthcoming Part II of this paper. Also, an application of the above ideas to certain problems involving Fourier-Stieltjes series will be developed there.

\section{REFERENCES}

1. C. V. Stanojević, Classes of $L^{1}$-convergence of Fourier and Fourier-Stieltjes series, Proc. Amer. Math. Soc. 82 (1981), 209-215.

2. Tauberian conditions for the $L^{1}$-convergence of Fourier series, Trans. Amer. Math. Soc. 271 (1982), 237-244.

3. G. A. Fomin, A class of trigonometric series, Mat. Zametki 23 (1978), 213-222.

4. A. Zygmund, Trigonometric series, Cambridge Univ. Press, New York, 1959.

5. G. A. Fomin, On the convergence in mean of Fourier series, Mat. Sb. 38 (1981), 231.

6. P. L. Butzer and R. J. Nesser, Fourier analysis and approximation. vol. I, Academic Press, New York, 1971.

Department of Mathematics, University of Maine at Orono, Orono, Maine 04469

Cedar Groves Estates, Rolla, Missouri 65401 